\title{
A histochemical study to identify nodal micro- metastasis in oral squamous cell carcinoma
}

\begin{abstract}
Background: Cancer cells tend to shed from the original tumor site and spread to another part of the body, group together and form a small collection of secondary tumors known as micro-metastatic tumor cells, frequently to lymph nodes in a predictable pattern often unobserved with advanced imaging techniques, that it can only be visible under microscope. Head and neck oral squamous cell carcinoma (HNSCC) has a significant potential for creating such micro-metastasis with higher incidence compared to clinically detected macro-metastasis.

Aim: The aim of the study is to detect micro-metastatic lymph node by tumor cells and evaluate an effective method by using routine hematoxylin and eosin stain, Modified Papanicolaou stain and Toluidine blue by serial sectioning.

Materials and method: A total of 60 lymph nodes retrospectively obtained from 10 patients diagnosed with oral squamous cell carcinoma who had undergone radical neck dissection, (i.e., three lymph nodes from both of Level I, II for each case), which initially identified as nonmetastatic by routine Hematoxylin and Eosin stain, were subjected to serial sectioning and re-evaluated for detection of micrometastasis. Results: Out of all the sections analyzed only 3 sections in level I and 2 sections in level II stained by Modified Papanicolaou stain showed positivity. Only 4 sections in level I lymph node and 3 sections in level II stained by toluidine blue showed positivity in detecting micro-metastasis. Conclusion: The present study concluded that special stains both toluidine blue and modified Papanicolaou stain are definitely valuable and sensitive in detecting micro-metastasis over Hematoxylin and Eosin stain.
\end{abstract}

Keywords: micro-metastasis, oral cancer, recurrence, serial section, toluidine blue

\author{
Volume 9 Issue 3 - 2018
}

\author{
Keerthi Narayan V,' Varsha Vimal Kumar, ${ }^{2}$ \\ Girish Hemadala, ${ }^{3}$ Sanjay Murgod, ${ }^{4}$ Sai Kavya \\ $M^{\prime}$ \\ 'Post Graduate Student, Department of Oral Pathology and \\ Microbiology, Rajarajeswari Dental College and Hospital, India \\ ${ }^{2}$ Senior Lecturer, Department of Oral Pathology and \\ Microbiology, Rajarajeswari Dental College and Hospital, India \\ ${ }^{3}$ Professor and Head, Department of Oral Pathology and \\ Microbiology, Rajarajeswari Dental College and Hospital, India \\ ${ }^{4}$ Professor, Department of Oral Pathology and Microbiology, \\ Rajarajeswari Dental College and Hospital, India
}

\begin{abstract}
Correspondence: Keerthi Narayan V, Rajarajeswari Dental College and Hospital, No: 14, Ramohalli Cross, Kumbalagodu, Bangalore, Karnataka-560074, India, Tel +9| 9|48326367, +9| 98948|8284,Email narayankrth55@gmail.com
\end{abstract}

Received: May 04, 2018 | Published: June II, 2018

\section{Introduction}

Oral cancer is the sixth most frequent cancer worldwide with a high prevalence in South Asia, accounting for one fourth of cancers in males and one tenth of cancers in females in India. ${ }^{1}$ Micro-metastasis to the lymph node is a vital prognostic factor as the innumerable changes brought about by these metastatic cells in the cervical lymph nodes is significantly important in survival, recurrence and also in making critical decisions concerning postoperative radiation treatment. ${ }^{2}$ Despite advanced imaging techniques, assessing the lymph node in the absence of clinical enlargement is challenging and may not be able to reveal small foci of tumor cells or micro-metastasis deposits. ${ }^{3}$ Studies have shown that routine analysis of lymph node by Hematoxylin and Eosin stain failed to observe up to $21 \%$ of diseased nodes and the risk of micro-metastasis is particularly high in oral cavity, oropharynx, hypopharynx and supraglottis. ${ }^{4}$ Approximately $10 \%$ of patients have been reported with regional recurrence with histologically diagnosed non metastatic lymph nodes resected suggesting that tumor cells were present but not identified. ${ }^{5}$

To detect micro-metastasis by expensive and technique sensitive methods such as Immunohistochemistry (IHC), Reverse Transcriptase Polymerase Chain Reaction (RT-PCR) of all the obtained lymph nodes is practically not feasible. Similarly the conventional one-section method for histopathological examination fails to spot out the small deposits. ${ }^{6-8}$ Hence, there is necessitating for the detection of micrometastasis by a method which is easily feasible and inexpensive. Thus, this study was carried out to identify few routinely available special stains such as Modified Papanicolaou stain and toluidine blue on serial sectioned lymph node sections to detect micro-metastasis which are routinely missed in single-section Hematoxylin and Eosin stain and to evaluate the efficient detection method.

\section{Materials and methods}

This retrospective study consisted of evaluation of serial sections of lymph nodes stained by Hematoxylin and Eosin stain, modified Papanicolaou stain and toluidine blue. It included a total of 60 paraffin embedded lymph node tissue blocks from 10 oral squamous cell carcinoma patients who had undergone neck dissection. 3 lymph nodes each from Level I, II respectively from every single case histopathologically diagnosed as non-metastatic were retrieved from the archives of Department of Oral Pathology and Microbiology of Rajarajeswari dental college and hospital, Bangalore, Karnataka.

Level I and Level II lymph nodes of sizes equal to/or greater than $1 \mathrm{~cm}$ at their greatest diameter were included in the study. These lymph nodes did not show any micro-metastatic tumor cells with one-section method. All histopathologically proved non metastatic 60 paraffin embedded lymph node tissue blocks were then sectioned for obtaining single sections and then the whole lymph node was cut at every $100 \mu \mathrm{m}$ till it was exhausted. The serial sectioned slides stained by routine Hematoxylin and Eosin, Modified Papanicolaou and toluidine blue stain were then analyzed for the detection of micro-metastasis and the results of all the three stains were compared to evaluate the efficacy of these three stains in detecting micro-metastasis. 


\section{Results}

The age of 10 patients ranged from fourth to eighth decade of life. Out of 10 patients in the study, 8 were females and only 2 were males with buccal mucosa being the predominant site of lesion with 9 patients. Of these 9 cases 6 lesions were exclusively located in buccal mucosa and 3 were in both buccal mucosa and Alveolus. Only one lesion was located in the Tongue. Five cases were of histological graded well differentiated and 5 were of moderately differentiated cases of oral squamous cell carcinoma.

Out of all the sections which were analyzed for detection of micrometastasis, only 3 sections in level I lymph node (Figure 1) (Figure 2) sections in level II stained by Modified Papanicolaou stain showed positivity in detecting micro-metastasis (Figure 2). Similarly only 4 sections in level I lymph node and 3 sections in level II stained by toluidine blue showed positivity in detecting micro-metastasis (Figure 3). Micro-metastatic tumor cells were observed in 5 cases in level I

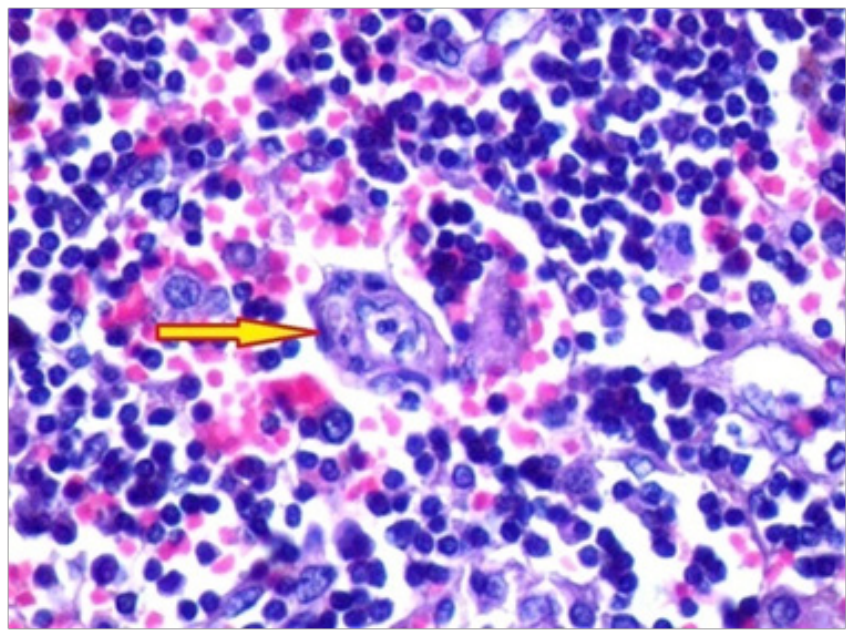

Figure I Photomicrograph reveals micro-metastatic tumor cells in level I lymph node stained by Modified Papanicolaou stain (x40) (arrows). Note high vascularity around the tumor cells.

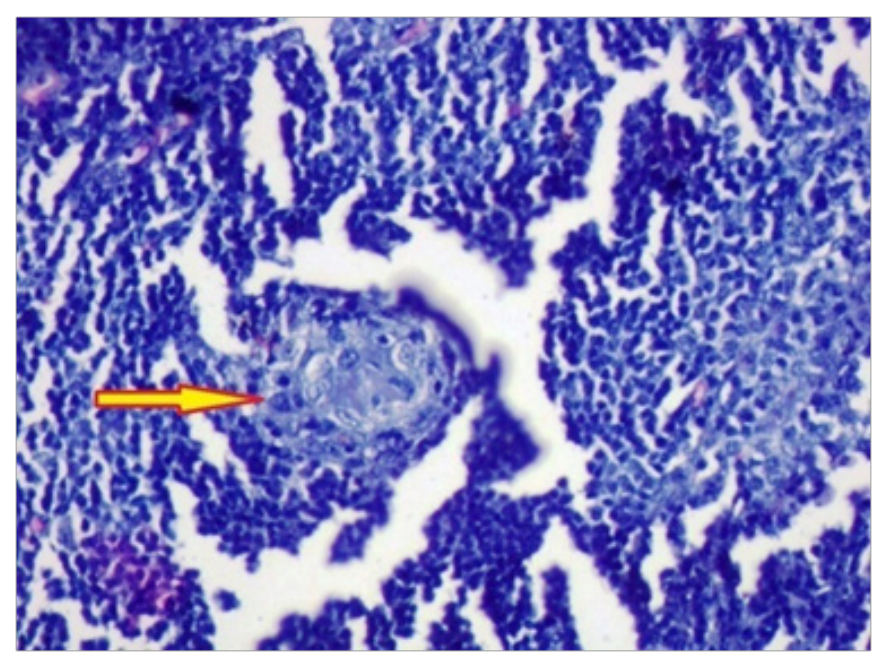

Figure 2 Photomicrograph reveals micro-metastatic tumor cells in level II lymph node stained by Modified Papanicolaou stain (x20) (arrows) lymph node and 5 cases in level II. Only 2 cases showed positivity in both level I and II. Based on the lymph nodes it was observed that 7 sections showed positivity in level I and 5 sections showed positivity in level II for micro-metastatic tumor cells (Table $1 \&$ Chart 1). Hematoxylin and Eosin stain were ineffective in detecting micrometastasis in all the sections examined (Figure 4).

Table I Table representing Lymph nodes showing Micro-Metastasis Positivity for Each staining type

\begin{tabular}{llll}
\hline $\begin{array}{l}\text { Lymph } \\
\text { nodes }\end{array}$ & $\begin{array}{l}\text { Hematoxylin } \\
\text { and eosin }\end{array}$ & $\begin{array}{l}\text { Modified } \\
\text { papanicolaou } \\
\text { stain }\end{array}$ & $\begin{array}{l}\text { Toluidine } \\
\text { blue stain }\end{array}$ \\
\hline Level I & 0 & 3 & 4 \\
Level 2 & 0 & 2 & 3 \\
Total & 0 & 5 & 7 \\
\hline
\end{tabular}

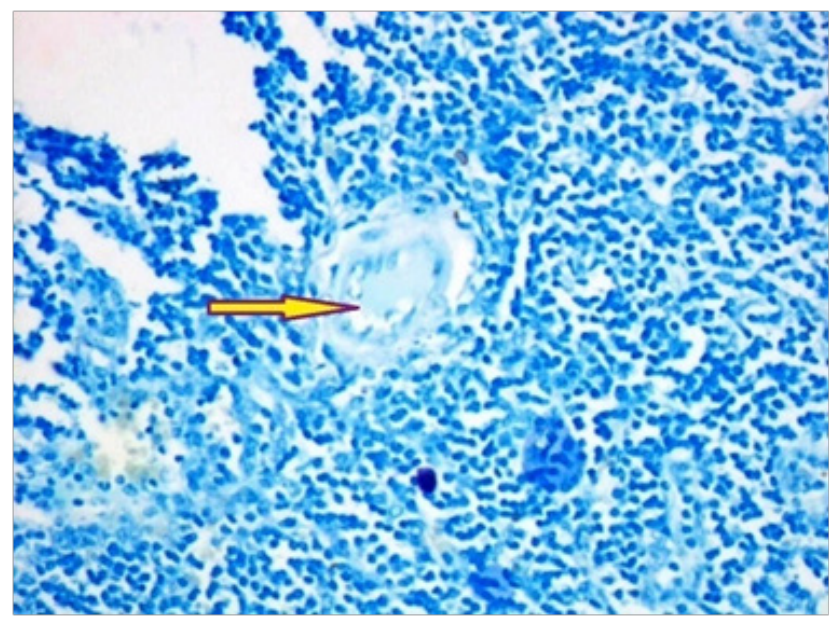

Figure 3 Photomicrograph reveals micro-metastatic tumor cells in level I lymph node stained by Toluidine stain (x20) (arrows)

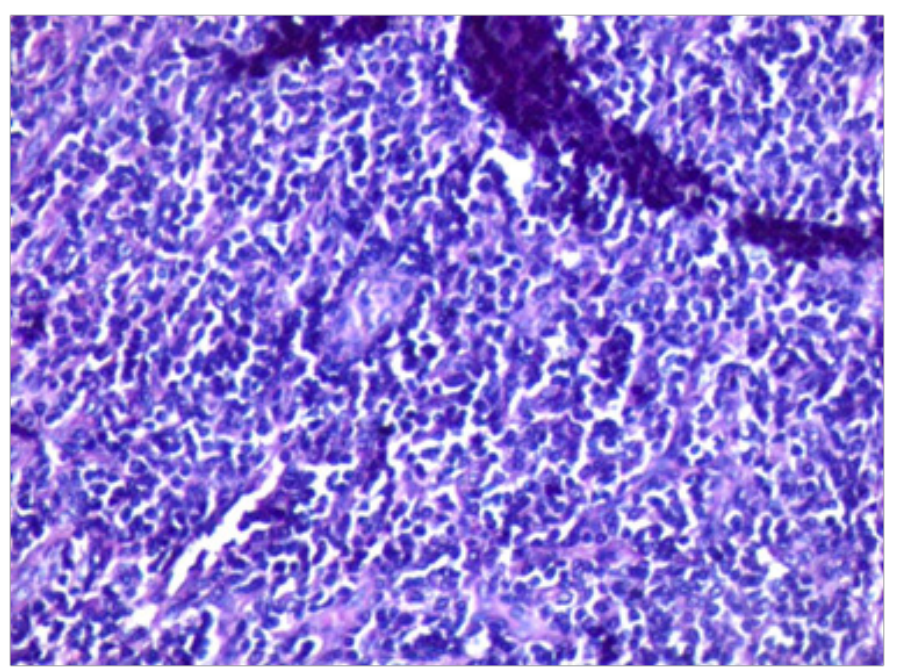

Figure 4 Photomicrograph of level I lymph node showing ineffective of Hematoxylin and Eosin in detecting nodal micro-metastasis $(x 20)$. 


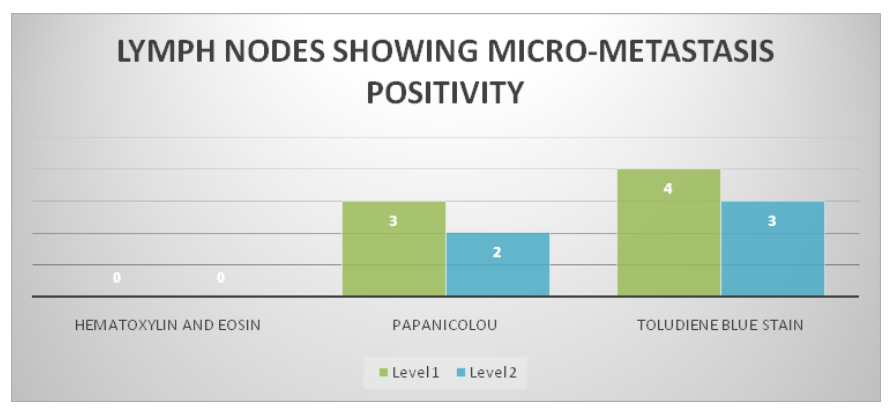

Chart I Bar chart representing Lymph nodes showing Micro-Metastasis Positivity for Each staining type.

Person's Chi-square distribution test was performed to look for the association between histological grading and micro-metastasis positivity. The statistical software package (SPSS) version 4.2 was used to perform the analysis. The $p$ value $(0.7469)$ was not significant at $\alpha=0.05$ (Table 2). The statistical value of this comparative analysis was found to be non-significant suggestive of non-association between histological grading and micro-metastasis positivity.

Table 2 Table showing the association between Histological grading and Micrometastasis Positivity

\begin{tabular}{lll}
\hline Histologic grading & $\begin{array}{l}\text { Positive } \\
\text { micrometastisis }\end{array}$ & $\begin{array}{l}\text { Negative } \\
\text { micrometastisis }\end{array}$ \\
\hline $\begin{array}{l}\text { I- well Differentiated } \\
\text { II- Moderately }\end{array}$ & 7 & 23 \\
differentiated & 5 & 25 \\
Total & 12 & 48 \\
*p=0.7469 & & \\
*statistically not significance at $\alpha=0.05$ & \\
\hline
\end{tabular}

\section{Discussion}

Squamous cell carcinoma of the head and neck (HNSCC) frequently metastasizes to the regional lymph nodes which are the primary location for capture of these tumor cells that have invaded the lymphatic system, hence the strongest predictor of disease prognosis and outcome. ${ }^{8,9}$ The presence of metastatic lymph nodes decreases survival rate by more than $50 \%$.Occult metastasis also known as micro-metastasis, are the microscopic foci of the metastasis in the nodes, which clinically fails to show detectable enlargement. ${ }^{10}$

Black et al defined micro-metastasis (MM) as tumor cells occupying less than $20 \%$ of the sectioned areas. ${ }^{11}$ De Mascarel et al described micro-metastasis as tumor deposits measuring less than $0.5 \mathrm{~mm} .^{12}$ Tumor deposits within the lymph nodes were classified and staged according to Union for International Cancer Control (UICC) which suggested micro-metastasis as metastasis occupying between $0.2 \mathrm{~mm}$ to $0.2 \mathrm{~cm}$ of the sectioned area. ${ }^{10}$ The isolated tumor cells (ITC) are the characteristic malignant cells which are seen in lymph nodes detected only by immuno histochemistry. The finding of isolated (disseminated or circulating) tumor cells (ITC) should be distinguished from micrometastasis (occult metastasis). A micro-metastasis occurs when there has been arrest and lodgment of tumor cells in the organ involved, extravasations, proliferation and stromal reaction. Thus, the diagnosis of micro-metastasis is possible only by histological examination. ${ }^{13,14}$

Anterior oral cavity drains to Level I lymph nodes while Level
II are drained by the posterior parts of the oral cavity. About $50 \%$ of oral cancer patients reveal lymph node metastasis. Hence in the present study Level I and Level II lymph nodes were included, as the metastasis of oral cancers primarily takes place in these lymph nodes levels. ${ }^{15}$

The standard surgical approach for oral squamous cell patients is resecting the primary tumor with the appropriate safety margins to ensure good local control followed by neck clearance for ensuring complete regional control. ${ }^{15,16}$

The use of classical radical neck dissection had decreased over the last 2 decades with the use of modified or selective neck dissection and also chemo-radiation as a non-surgical option. Following such classical radical neck dissection procedures in high risk, poorly differentiated cases frequently leads to a significant functional disturbance of the accessory nerve and cosmetic morbidity of the affected patients. Recurrence in these cases increases the chance of morbidity significantly. This illustrates the importance of detection of even a single micro-metastasis tumor cell, which has a significant role in prognosis and survival of the patient. ${ }^{3}$

Studies on micro-metastasis have been carried in gastric cancers, breast cancers, colorectal cancers, carcinomas of other sites and their results have shown that the detection of micro-metastasis has a significant difference in the recurrence and survival rate. ${ }^{12,17}$

Čelakovský et al. ${ }^{18}$ recommended Elective neck dissection or radiotherapy of the neck should be considered in patients with high risk of occult metastases or micro-metastasis. ${ }^{18}$ Thakare et al. ${ }^{5}$ in their study observed micro-metastatic carcinomatous cells in $\mathrm{H}$ and E staining of frozen section (FS) in 18 lymph nodes (54\%) and in 19 lymph nodes (57\%) in step-serial sectioned (SS) H and E-stained sections. ${ }^{5}$ Thome JA et al. ${ }^{19}$ results showed that intra-operative FS is a highly accurate and sensitive method to detect macro-metastasis. However, it is inaccurate in the detection of micro-metastasis. ${ }^{19}$

In the view of crucial role of occult LN metastasis in prognosis and survival of OSCC patients with N0 neck Dhawan et al. ${ }^{9}$ recommended diagnostic tools such as IHC staining, particularly with pan-CK (AE1/ AE3), combined with SS should be preferred over conventional methods as they result in upstaging, thus sparing the low-risk patients the morbidity of unnecessary treatment. ${ }^{9}$

Histopathological analysis of neck dissection specimens is usually performed on several 3-4- $\mu \mathrm{m}$ sections from each lymph node; micrometastasis can be easily missed on routine light microscopy. De Mascarel et al. ${ }^{12}$ concluded Serial Macroscopic sectioning (SMS) does not require excessive work and micro-metastasis do have a prognostic significance. ${ }^{12}$ In breast cancers, Nasser et al employed serial sectioning at $150 \mu \mathrm{m}$ intervals and he detected a $31 \%$ significance rate. ${ }^{20} \mathrm{MC}$ Guckin et al. ${ }^{21}$ did serial sectioning at $100 \mu \mathrm{m}$ intervals and detected a $25 \%$ significance rate. Thus, it can be seen that the detection rate varied from $9-33 \%{ }^{21}$

Prakash et al. ${ }^{10}$ revealed the detection of micro-metastasis by the serial sectioning method was $2.03 \%$. He detected micro-metastasis in only two nodes out of 119 nodes of the 16 cases, of which 96 were from node negative and 16 were from node positive cases. ${ }^{10}$ In the present study out of 60 histological proven negative lymph nodes from 10 cases showed micro-metastasis positivity in only 12 nodes and 48 nodes were negative for detection. 
Dhawan et al. ${ }^{9}$ in a study on application of combination of SS and IHC using pan-CK (AE1/AE3) revealed the presence of MM and ITC in $2.25 \%$ of the Lymph nodes diagnosed as negative on routine H\&E examination. The detection of these occult metastatic deposits resulted in upstaging of $33.33 \%$ of the patients. ${ }^{9}$

Thakare et al. ${ }^{5}$ showed 19 lymph nodes (57\%) in step-serial sectioned (SS) $\mathrm{H}$ and E-stained sections in contrast to our study where $H \& E$ stain failed to yield any detectable positive sections. The probable reason for such difficulty experienced in this study for differentiating a single cell or a group of malignant cells which invaded the lymph nodes in the $\mathrm{H}$ and $\mathrm{E}$ sections owing to closely resemblance of the endothelial cells and the histiocytes with the malignant squamous cells. ${ }^{3,5}$

Extensive research of English literature have shown numerous studies on detection of micro-metastasis by cryosection, conventional $\mathrm{H}$ and $\mathrm{E}$ staining, immunohistochemistry and RT-PCR analysis but has not revealed any studies exclusively on detection of micro-metastasis in oral squamous cell carcinoma by incorporating special stains, in particular for keratin which adds to the uniqueness of this study.

The special stains used in the present study highlightened even small foci of evident epithelial differentiation by absence of hyperchromatism, difference in size, shape and keratin formation, frequently missed in routine Hematoxylin and Eosin staining by orange to pink color to the cytoplasm of the metastatic epithelial cells. $^{22}$

Various studies have shown keratins, the major constituent of the cytoskeleton of all epithelial cells can be used as a marker protein to detect metastatic epithelial cells or micro-metastasis. Preethi S et al. ${ }^{23}$ in a comparative study illustrated that Papanicolaou stains more clearly demonstrated key morphological features associated with keratinization in tissue sections than the routine Hematoxylin and Eosin stained tissue sections..$^{23}$

Papanicolaou stain, a differential chromatic stain being inexpensive, easily available, containing Harris's Hematoxylin, Orange G6, Eosin-Azure commonly used for cytological smears has been used to stain paraffin embedded tissue sections for demonstration of keratin in the present study. Superficial cells with high content of keratin stain yellow-orange tinge and parabasal cells stain green to blue in color. ${ }^{24,25}$

Mumtaz WR et al. ${ }^{3}$ observed that Papanicolaou stain was the only stain useful in detecting micro-metastasis which accounted to $7 \%$ of non metastatic lymph node sections. Out of the 100 sections which were analyzed only 7 sections showed positivity in detecting micrometastasis. ${ }^{3}$ Similarly in the present study Modified Papanicolaou stain showed 5 sections that retained positive for micro-metastasis. Of this, 3 are level I lymph node tissue sections and 2 are from level II lymph node tissue sections.

The toluidine blue stain, similar to the Papanicolaou smear for cervical lesions, is a useful, simple screening procedure for vulvar surfaces. It aids in the detection, demonstration and follow-up of vulvar lesions. ${ }^{26,27}$ Toluidine blue, a vital acidophilic metachromatic die that stains acidic tissue components being simple, cost effective and easy to perform also has an application in staining tissue section. ${ }^{28,29}$ Toluidine blue selectively stained the acidic tissue structures (sulfates, carboxylates, and phosphate radicals); this affinity mechanism of differentiation from rest of cells was still not clearly understood. ${ }^{29-31}$

Toluidine blue is used mostly for staining of frozen sections of basal cell carcinomas (BCCs) during surgery due to its ability to accentuate the tumor's mucopolysaccharide-rich stroma. The same stromal reaction also can be observed around OSCC. It is considered very valuable in its ability to emphasize delicate cords and sparse clusters of cells associated with infiltrative neoplasm's, such as infiltrative BCC, infiltrative SCC, and microcystic adnexal carcinoma. ${ }^{32}$

As for keratin, positive staining was recently shown to be in relation to disulfide groups on cysteic acid formation under oxidation. ${ }^{33}$ Prunieras demonstrated a red metachromatic color in the outer layer of the hair root in a normal hair containing skin stained with Toluidine Blue for metachromasia, but on oxidization and restaining in the same way does not show any metachromasia. ${ }^{34}$ In the present study Toluidine blue method demonstrated 7 positive sections with micrometastasis. Of these, 4 are level I lymph node tissue sections and 3 are level II sections. Since very few studies have been done using toluidine blue stain in tissue sections, there is scarcity of published data.

Alkaisi et al. ${ }^{35}$ in multivariate analysis on the most important prognostic factors such as tumor depth followed by pattern of invasion, tumor site, and degree of differentiation concluded degree of differentiation as a reliable factor to predict lymph node micrometastasis in conjunction with other factors. ${ }^{35}$ In contrast, there is no association was observed between histological grading and micrometastasis positivity in our study.

\section{Limitations}

This study has few limitations like the sample size, was too small to derive a conclusion statistically. Also, the hypothesis that whether the two special stains mentioned in the present study can be used to detect micro-metastasis is arguable. Hence, more wide-ranging studies are obligatory in this field.

\section{Conclusion}

The present study concluded that special stains both toluidine blue and modified Papanicolaou stain are definitely valuable and sensitive in detecting micro-metastasis over Hematoxylin and Eosin stain by serial sectioning technique. Detection of micro-metastasis in oral squamous cell carcinoma patients post surgically is unquestionably advantageous for the patient as it modifies the treatment plan in terms of both radiotherapy and chemotherapy.

\section{Acknowledgements}

I am grateful to Dr. Prakash, Onco-surgeon for his wholehearted support for this study and also I would like to acknowledge Mrs. Mrinalini Kabbur, Statistician for her continuous support to overcome methodical difficulties in the study.

\section{Conflict of interest}

Author declares that there is no conflict of interest.

\section{References}

1. Agarwal P, Chaudhary M, Patil S, et al. Diagnostic accuracy of various methods to detect lymph node metastasis in oral squamous cell carcinoma. J Evolution Med Dent Sci. 2014;3(22):6003-10. 
2. Ambrosch P, Brinck U. Detection of nodal micrometastases in head and neck cancer by serial sectioning and immunostaining. Oncology (Williston Park). 1996;10(8):1221-26.

3. Mumtaz WR, Hedge V, Yadav N. Micrometastasis detection using special stains in nodal tissues of oral squamous cell carcinoma- A histochemical study. Journal of Clinical and Diagnostic Research. 2016;10(12):ZC23ZC26.

4. Shivers SC, Wang X, Li W, et al. Molecular staging of malignant melanoma: Correlation with clinical outcome. JAMA. 1998;280(16):1410-15.

5. Thakare E, Gawande M, Chaudhary M, et al. Detection of micrometastasis in lymph nodes of oral squamous cell carcinoma: A comparative study. $J$ Oral Maxillofac Pathol. 2013;17(3):374-380.

6. Schoenfeld A, Luqmani Y, Smith D, et al. Detection of breast cancer micrometastases in axillary lymph nodes by using polymerase chain reaction. Cancer Res. 1994;54(11):2986-90.

7. Ferris RL, Xi L, Raja S, et al. Molecular staging of cervical lymph nodes in squamous cell carcinoma of the head and neck. Cancer Res. 2005;65(6):2147-56.

8. Becker MT, Shores CG, Yu KK, et al. Molecular assay to detect metastatic head and neck squamous cell carcinoma. Arch Otolaryngol Head Neck Surg. 2004;130(1):21-7.

9. Dhawan I, Sandhu SV, Bhandari R, et al. Detection of cervical lymph node micrometastasis and isolated tumor cells in oral squamous cell carcinoma using immunohistochemistry and serial sectioning. J Oral Maxillofac Pathol. 2016;20(3):436-44.

10. Prakash AR, Kumar GS, Shetty P. A Study on the detection of micrometastasis in the cervical lymph nodes of oral squamous cell carcinomas by serial sectioning. J Clin and Diagn Res. 2011;5(1):78-81.

11. Black RB, Roberts MM, Stewart HJ, et al. The search for occult metastases in breast cancer: does it add to established staging methods. Aust $N \mathrm{ZJ}$ Surg. 1980;50(6):574-79.

12. de Mascarel I, Bonichon F, Coindre JM, et al. Prognostic significance of breast cancer axillary lymph node micrometastases assessed by two special techniques: reevaluation with longer follow-up. $\mathrm{Br} J$ Cancer. 1992;66(3):523-27.

13. Hermanek P, Hutter RV, Sobin LH, et al. International Union against Cancer. Classification of isolated tumor cells and micrometastasis. Cancer. 1999;86(12):2668-73.

14. Todo Y, Kato H, Okamoto K, et al. Isolated tumor cells and micrometastasis in regional lymph nodes in stage I and II endometrial cancer. J Gynecol Oncol. 2016;27(1):e1

15. Teymoortash A, Werner JA. Current advances in diagnosis and surgical treatment of lymph node metastasis in head and neck cancer. GMS Curr Top Otorhinolaryngol Head Neck Surg. 2012;11:04.

16. Cheng PT, Hao SP, Lin YH, et al. Objective comparison of shoulder dysfunction after three neck dissection techniques. Ann Otol Rhinol Laryngol. 2000;109(8 Pt 1):761-66.

17. Dowlatshahi K et al. Lymph node micrometastases from breast carcinoma: Reviewing the dilemma. Cancer. 1997;80(7):1188-97.
18. Celakovsky P, Kalfert K, Smatanova K, et al. Detection of cervical lymph node micrometastasis in patients with squamous cell carcinoma of the oral cavity, pharynx and larynx. J Acta Medica. 2015;58(2):62-65.

19. Thomé, JA, Mendiburu CF, Palma CF, et al. Intraoperative frozen section assessment in the evaluation of axillary sentinel lymph node in breast cancer. J Bras Patol Med Lab. 2012;48(5);369-373.

20. Nasser IA. Occult axillary lymph node metastases in node negative breast carcinoma. Hum Pathol. 1993;24(9):950-7.

21. McGuckin MA. Occult axillary node metastases in breast cancer: their detection and prognostic significance. Br. J Cancer. 1996;73(1):88-95.

22. Johnson Pergy L, Klein Morris N. Application of Papanicolaou stains to paraffin section. Stain Technol. 1956;31(5):223-25.

23. Preethi S, Sivapathasundharam B. Will modified Papanicolaou stain be the new stain for keratin. Journal of Histotechnology. 2015;38(1):9-13.

24. Elzay RP. A modification of Papanicolaou exfoliative cytology stains to demonstrate keratin in paraffin-block tissue sections. Oral Surg Oral Med Oral Pathol. 1983;56(1):51-53.

25. Rao RS, Patil S, Majumdar B, et al. Comparison of special stains for keratin with routine hematoxylin and eosin stain. $J$ Int Oral Health. 2015;7(3):1-5.

26. Sridharan G, Shankar AA. Toludine blue: a review of its chemistry and clinical utility. J Oral Maxillofac Pathol. 2012;6(2):251-255.

27. Hall DJ, Hart WG. Lesions of the vulva. J Fam Pract. 1984;18:129-133.

28. Lingen MW, Kalmar JR, Karrison T, et al. Critical evaluation of diagnostic aids for the detection of oral cancer. Oral Oncol. 2008;44(1):10-22.

29. Chhabra N, Chhabra S, Sapra N. Diagnostic Modalities for Squamous Cell Carcinoma: An Extensive Review of Literature-Considering Toluidine Blue as a Useful Adjunct. J. Maxillofac. Oral Surg. 2015;14(2):188-200.

30. Epstein JB, Scully C, Spinelli J. Toluidine blue and Lugol's iodine application in the assessment of oral malignant disease and lesions at risk of malignancy. J Oral Pathol Med 1992;21(4):160-163.

31. Gandolfo S, Pentenero M, Broccoletti R, et al. Toluidine blue uptake in potentially malignant oral lesions in vivo: clinical and histological assessment. Oral Oncol. 2006;42(1):89-95.

32. Huphreys TR, Nemeth A, McGrevey S, et al. A pilot study comparing toluidine blue and hematoxylin and eosin staining of basal cell and squamous cell carcinoma during Mohs surgery. Dermatol Surg. 1996;22(8):693-7.

33. Adams CWM, Sloper JC. The hypothalamic elaboration of posterior pituitary principles in man, the rat and dog. Histochemical evidence derived from a perfomic acid-alcian blue reaction for cystine. $J$. Endocrinol. 1956;13(3):221-228.

34. Prunieras M. PAB: Stain for Tissue, Keratin and Fungi. Journal of Investigative Dermatology. 1959;66:309-314.

35. Alkaisi A, Zaidan HA, Al Kabtan IAH. The Predictive Value of Tumor Depth for Cervical Lymph Node Metastasis in Oral Squamous Cell Carcinoma; Prospective and Retrospective Study in Iraq. J Cancer Sci Ther. 2014;6:253-257. 